\title{
Biopelículas de Gelana Cargadas con Nanopartículas de Plata: Propiedades Físico Mecánicas, Antimicrobianas y de Barrera
}

\author{
Rafael E. González, Arnulfo Tarón y Jaime Pérez \\ Facultad de Ingeniería. Departamento de Ciencias de los Alimentos. Grupo de Investigación en \\ Biotecnología de Alimentos y Educación (GIBAE). Universidad de Cartagena, Av. Del Consulado, Calle \\ $30 \mathrm{~N}^{\circ}$ 48-157., Cartagena de Indias - Colombia (e-mail: rgonzalezc1@unicartagena.edu.co)
}

Recibido Abr. 28, 2017; Aceptado Jul. 3, 2017; Versión final Ago. 8, 2017, Publicado Dic. 2017

\begin{abstract}
Resumen
Se elaboraron biopelículas a base de gelana de bajo acilo cargadas con nanopartículas de plata (AgNPs) evaluando sus propiedades físico-mecánicas, antimicrobianas y de barrera, después de un almacenamiento de 3 semanas. La actividad antimicrobiana fue evaluada frente a bacterias de origen alimentario (Escherichia coli y Staphylococcus aureus) y dos hongos (Aspergillus sp y Penicillium sp). Los resultados permitieron determinar que el tiempo de almacenamiento influye en todas las propiedades evaluadas. Al incrementar el tiempo de almacenamiento se apreció una disminución en la resistencia a la tensión y en la transparencia. Además se presentó un incremento en el poder antimicrobiano y la permeabilidad al vapor de agua. Este comportamiento probablemente obedece a la lixiviación de iones plata, lo cual disminuye el número de enlaces presentes en la matriz polimérica. Tales resultados son prometedores y sugieren la posibilidad de utilizar biopelículas a base de gelana de bajo acilo como sistema de vehiculización de AgNPs para su liberación gradual manteniendo un efecto antimicrobiano sostenido.
\end{abstract}

\section{Biofilms of Gellan Loaded with Silver Nanoparticles: Physico Mechanical, Antimicrobials and Barrier Properties}

\begin{abstract}
Biofilms based on low acyl gellan loaded with silver nanoparticles (AgNPs) were tested, evaluating their physical-mechanical, antimicrobial and barrier properties after 3 weeks of storage. The antimicrobial activity was evaluated against food-borne bacteria (Escherichia coli and Staphylococcus aureus) and two fungi (Aspergillus $s p$ and Penicillium $s p$ ). The results showed that the storage time influence all the evaluated properties. Increasing the storage time decreases the tensile strength and transparency values. Also, the antimicrobial power and the water vapor permeability increased. This behavior is probably due to the leaching of silver ions, which decreases the number of bonds present in the polymer matrix. Such results are promising and suggest the possibility of using biofilms based on low acyl gellan as a system for the vehiculation of AgNPs for their gradual release while maintaining a sustained antimicrobial effect.
\end{abstract}

Keywords: biofilms; gellan gum; silver nanoparticles; permeability; tensile strength 


\section{INTRODUCCIÓN}

A nivel mundial, cerca de un tercio de los alimentos producidos para consumo humano se descomponen como consecuencia de factores físicos, químicos y biológicos. Los golpes y las magulladuras causan un deterioro en la textura de las diferentes matrices alimentarias, mientras que por el crecimiento microbiano se producen olores desagradables y cambios en la coloración y textura. Además, algunos microorganismos pueden producir toxinas representando un riesgo importante para la salud de los consumidores (Corrales et al., 2014). Por lo anterior, existe la necesidad de encontrar métodos que contribuyan en la conservación de la textura, así como el control de microorganismos patógenos durante el procesamiento, empacado y/o distribución de alimentos. Las biopelículas (BP) han surgido en las últimas décadas como una alternativa para sustituir parcial o completamente los materiales poliméricos sintéticos. En este sentido, el desarrollo de BP poliméricas se ha vislumbrado como una opción interesante. Dentro de los materiales poliméricos la goma gelana ha sido utilizada con buenos resultados (González et al., 2017a).

La goma gelana es un tetrasacárido lineal aniónico compuesto por 1-3 $\beta$ D glucosa, 1,4 $\beta$ D ácido glucurónico, $1,4 \beta$ D glucosa, $\alpha-L$ ramnosa, que en su forma nativa se conoce como gelana de alto acilo (GAA) porque presenta grupos acetato y glicerato en su residuo de glucosa. Cuando la GAA es expuesta a un fuerte tratamiento con álcali a elevadas temperaturas, los grupos acilo son hidrolizados y la gelana de bajo acilo (GBA) es obtenida. La GBA se caracteriza por formar geles más firmes que la GAA (González et al., 2012) y además, por su naturaleza aniónica puede formar geles en presencia de cationes. Las BP en general son utilizadas como una barrera a la migración de humedad y gases, aunque también pueden actuar como acarreadores de ingredientes de importancia como compuestos activos, saborizantes, colorantes y agentes antimicrobianos, que ayudan a extender la vida útil de las matrices alimentarias en las que son aplicadas (Kokoszka et al., 2010).

Dentro de las múltiples aplicaciones de las BP, la de mayor interés para la industria agroalimentaria, es la producción de empaques modificados con compuestos metálicos. Los metales provenientes de fuentes minerales han sido utilizados en forma de sales, óxidos y coloides, debido a sus propiedades antimicrobianas. De los metales, la plata exhibe un alto grado de toxicidad contra microorganismos (bacterias, hongos y virus) y una menor toxicidad contra células mamíferas (Rai et al., 2009). Además, el uso de este metal presenta ventajas como como una mayor estabilidad térmica, baja volatilidad y bajos costos de producción (MartínezAbad et al., 2014). La plata en su estado metálico es un material inerte, pero puede reaccionar con la humedad produciendo iones de plata. Recubrimientos conteniendo nanopartículas de plata (AgNPs) confieren excelentes propiedades de conservación de alimentos (Metak et al., 2015) debido a la actividad microbicida que exhiben las AgNPs así como su elevada estabilidad.

La plata es uno de los aditivos con características antimicrobianas mayoritariamente utilizado en diversos materiales poliméricos como el agar (Ghosh et al., 2010; Rhim et al., 2013), alcohol de polivinilo (Bryaskova et al., 2010; Sedlarik et al., 2010), gelatina (Kanmani y Rhim, 2014) y mezclas de quitosan-celulosa (Lin et al., 2015), almidón-arcilla (Abreu et al., 2015) o quitosan-glutaraldehido (Vimala et al., 2011) para aplicaciones de empacado de alimentos (Martínez-Abad et al., 2012). Sin embargo, no hay casi estudios concernientes al desarrollo de BP a base de GBA cargadas de AgNPs y su comportamiento físico-mecánico. La resistencia a la tensión (RT) y la elongación al quiebre (EQ) son las principales propiedades físico-mecánicas mayoritariamente evaluadas en los materiales tendientes a ser utilizados como empaques de matrices alimentarias; ya que estos parámetros son medidas apropiadas para establecer la resistencia y la flexibilidad del material (Kurt y Kahyaoglu, 2014), y por tanto permiten seleccionar los mejores componentes para fabricar $\mathrm{BP}$ resistentes a esfuerzos externos que simulen el proceso de empacado

Por consiguiente, el objetivo del presente estudio fue determinar el efecto de las AgNPs sobre las propiedades físico-mecánicas, antimicrobianas y de barrera a través del tiempo de almacenamiento de biopelículas a base de gelana de bajo acilo.

\section{MATERIALES Y MÉTODOS}

La goma gelana de bajo (GBA) y la glucono $\delta$ lactona de grado alimenticio fueron obtenidas de Modernish pantry (EEUU). El $\mathrm{AgNO}_{3}$ así como el $\mathrm{CaCO} 3$ y el glicerol fueron suministrados por Merck-Colombia.

\section{Elaboración de las biopelículas}

Las dispersiones formadoras de BP-AgNPs fueron obtenidas por la reducción de $\mathrm{AgNO}_{3}$ usando luz ultravioleta (Monge, 2009). $20 \mathrm{mM}$ de $\mathrm{AgNO}_{3}$ fueron adicionados a las dispersiones $0.7 \% \mathrm{v} / \mathrm{v}$ de GBA. A cada mezcla se le adicionaron $10 \mathrm{mM}$ de $\mathrm{Ca}^{++}$y se mantuvieron a $90^{\circ} \mathrm{C}$ por 20 min bajo agitación constante y radiación UV hasta cambio de coloración debido a la formación de AgNPs. Luego, se adicionó el glicerol al 
$12 \%(p / v)$. La reducción del nitrato de plata a AgNPs fue monitoreado por espectrofotometría (Thermo Thomson Gold Spectrumlab 54) a $420 \mathrm{~nm}$. Finalmente, se adicionó glucono $\delta$ lactona con el fin de disminuir el pH hasta un valor de 4.0 y acelerar el proceso de gelificación de la GBA. Para la formación de las BP fueron vertidos volúmenes específicos de cada solución anterior en cajas de Petri. El secado se realizó en estufa a $30{ }^{\circ} \mathrm{C}$ durante 45 horas asegurando uniformidad de las BP. Igualmente, BP control fueron elaboradas sin la adición de $\mathrm{AgNO}_{3}$. Todas las BP elaboradas fueron almacenadas en un ambiente controlado durante tres semanas y se les determinaron las propiedades mecánicas (RT y EQ), de barrera (PVA) y antimicrobianas al inicio (semana 0 ) y al final del almacenamiento (semana 3 ).

\section{Propiedades mecánicas}

La resistencia a la tensión (RT) y la elongación al quiebre (EQ) fueron determinadas en un texturometro Shimadzu modelo EZ-Test EZ-S utilizando muestras rectangulares de $3 \times 6 \mathrm{~cm}$. Una geometría de mordazas fue utilizada con una velocidad de separación de $30 \mathrm{~mm} / \mathrm{min}$. Repeticiones de diez fueron llevadas a cabo a cada muestra y se reportan los promedios aritméticos y la desviación estándar. La RT en cada caso fue calculada dividiendo la carga máxima (N) por el área transversal $\left(\mathrm{m}^{2}\right)$ de las muestras como se muestra en la ecuación 1.

$\mathrm{RT}(\mathrm{MPa})=\frac{\mathrm{Fmax}}{\mathrm{A}}$

Donde $F_{\max }$ es la fuerza máxima que se requiere para romper la muestra $(\mathrm{N})$ y $\mathrm{A}$ es el área transversal de las $\mathrm{BP}\left(\mathrm{m}^{2}\right)$ que resulta de multiplicar el ancho por el espesor de la muestra. En cuanto al porcentaje de EQ fue estimado mediante la ecuación 2.

$\mathrm{EQ}=\frac{l_{\max }}{l_{\mathrm{o}}} \times 100$

Donde $I_{\max }$ es la elongación en milímetros $(\mathrm{mm})$ al momento de la ruptura, mientras que lo es longitud inicial $(\mathrm{mm})$.

\section{Espesor y Permeabilidad del vapor de agua (PVA)}

El espesor de las muestras fue determinado mediante un micrómetro de $0.01 \mathrm{~mm}$ de exactitud, seis puntos diferentes de cada muestra fueron medidos reportando los promedios aritméticos con sus respectivas desviaciones estándar. La PVA fue determinada gravimétricamente siguiendo el método propuesto por la ASTM E96/E96M, 2010 con un porcentaje de humedad relativa del $75 \%$ a una temperatura promedio de $30^{\circ} \mathrm{C} \pm 1{ }^{\circ} \mathrm{C}$ basándose en la ecuación 3 .

$P V A=\left[\frac{W}{t}\right] \cdot\left[\frac{\mathrm{x}}{\Delta \mathrm{P} \cdot \mathrm{A}}\right]$

Donde, $W$ es el cambio de peso por acción de la absorción de agua (g); t es el tiempo en días; $x$ es el espesor en $(\mathrm{mm}) ; A$ corresponde al área de la BP $\left(\mathrm{m}^{2}\right) ; \Delta \mathrm{P}$ es la diferencia de presiones parciales a través de la biopelículas. La $\triangle \mathrm{P}$ fue estimada mediante la ecuación 4 :

$\Delta P=S *\left(R_{1}-R_{2}\right)$

Donde, $\mathrm{S}$ es la presión del vapor de agua saturada a $25^{\circ} \mathrm{C}(3166 \mathrm{kPa}) ; \mathrm{R}_{1}$ es la humedad relativa en el desecador (0.82); $R_{2}$ es la humedad relativa en el interior (0.18) expresada en fracciones.

\section{Transparencia}

Para la estimación de este parámetro, muestras de las BP fueron cortadas en rectángulos $(2 \times 3 \mathrm{~cm})$ y colocadas en la celda del espectrofotómetro (Thomson Gold Spectrumlab 54). Fue fijada una incidencia de longitud de onda a $560 \mathrm{~nm}$ utilizando el aire como como referencia. Los valores de transparencia son obtenidos al multiplicar el espesor de cada muestra por la absorbancia obtenida (González et al., 2017a).

\section{Análisis antimicrobiano}

Cultivos de Escherichia coli, Staphylococcus aureus, Aspergillus spp y Penicillium spp fueron aislados por el Laboratorio de Microbiología de la Universidad de Cartagena (campus piedra de Bolívar). Las pruebas de actividad antimicrobiana se realizaron por el método de difusión en placa. Las bacterias aisladas fueron sembradas de forma masiva en las cajas de Petri conteniendo el agar específico para bacterias u hongo 
respectivamente. Seguidamente, muestras redondas (4 mm diámetro) de BP control (sin AgNPs) y BP-AgNPs y fueron dispuestos en las respectivas cajas de Petri e incubadas durante 24 horas a $37^{\circ} \mathrm{C}$ para el caso de bacterias y 3 días a $25^{\circ} \mathrm{C}$ para el caso de hongos. El grado de biocontrol fue calculado midiendo los diámetros de inhibición de las BP. Los ensayos fueron realizados por triplicado y los promedios aritméticos son los reportados para cada análisis.

\section{Análisis de Datos}

Los valores de las propiedades evaluadas (RT, EQ, PVA y efecto antimicrobiano) fueron sometidas a un análisis normal de varianza (ANOVA un factor) utilizando la prueba tukey ( $5 \%$ de significancia) mediante el programa de computo SPSS (Statistical Package for the Social Sciences) versión 17.0 para Windows (SPSS Inc. Chicago, IL, USA).

\section{RESULTADOS Y DISCUSIÓN}

Los resultados se presentan en tres secciones: (i) Resistencia a la tensión y elongación al quiebre; (ii) Espesor, transparencia y permeabilidad al vapor de agua de las biopelículas y (iii) Actividad antimicrobiana

\section{Resistencia a la tensión y elongación al quiebre de las Biopelículas}

En la figura 1 se aprecia que al adicionar las AgNPs se obtienen los mayores valores de RT (45.43 MPa) inicialmente; sin embargo, este valor disminuye hasta $32.21 \mathrm{MPa}$ después de tres semanas de almacenamiento. Con respecto a las muestras control, no se presentaron diferencias significativas $(\mathrm{P}<0.05)$ encontrándose valores de 23.81 y $22.97 \mathrm{MPa}$ al comienzo y al finalizar el periodo de almacenamiento (tres semanas) respectivamente. No obstante, estos valores de RT son menores a los publicados por Liling et al., (2016), quienes encontraron valores de $134.580 \mathrm{MPa}$ en BP de alginato utilizando $\mathrm{Ca}^{++}$como agente entrecruzante, este resultado podría ser consecuencia de un menor reforzamiento en el entrecruzamiento de los iones de plata con las hélices de gelana. Resultados similares fueron reportados por González et al., (2017b) estos autores encontraron valores de resistencia de 51.24 MPa en BP de GBA entrecruzadas con 30 $\mathrm{mM}$ de iones de calcio. De forma general la presencia de AgNPs incrementa significativamente $(P<0.05)$ los valores de RT. Varios autores (Cano et al., 2016; Rhim et al., 2013; Shameli et al., 2010) también han obtenido resultados similares, los cual ha sido atribuido a la adsorción de la plata en las cadenas poliméricas, en línea con las interacciones de van der Waals entre los grupos hidroxilo y la carga parcial positiva en la superficie de las nanopartículas de plata.

Igualmente, es importante mencionar que al haber utilizado $\mathrm{Ca}^{++}$como agente entrecruzante en la elaboración de las BP se pudo presentar una sobresaturación de los sitios activos de la cadena polimérica, conllevando esto a un efecto plastificante de las especies de plata en la matriz (Cano et al., 2016), y por consiguiente se obtuvieron bajos valores de RT. En este sentido, un posible incremento en la fuerza iónica en el medio acuoso cuando se incrementa la concentración de nitrato de plata, implica la reducción en el volumen libre de las macromoléculas antes de la formación de las BP. Dicho efecto reduce las fuerzas intermoleculares entre las cadenas poliméricas originando unos geles con atributos de mayor debilidad.
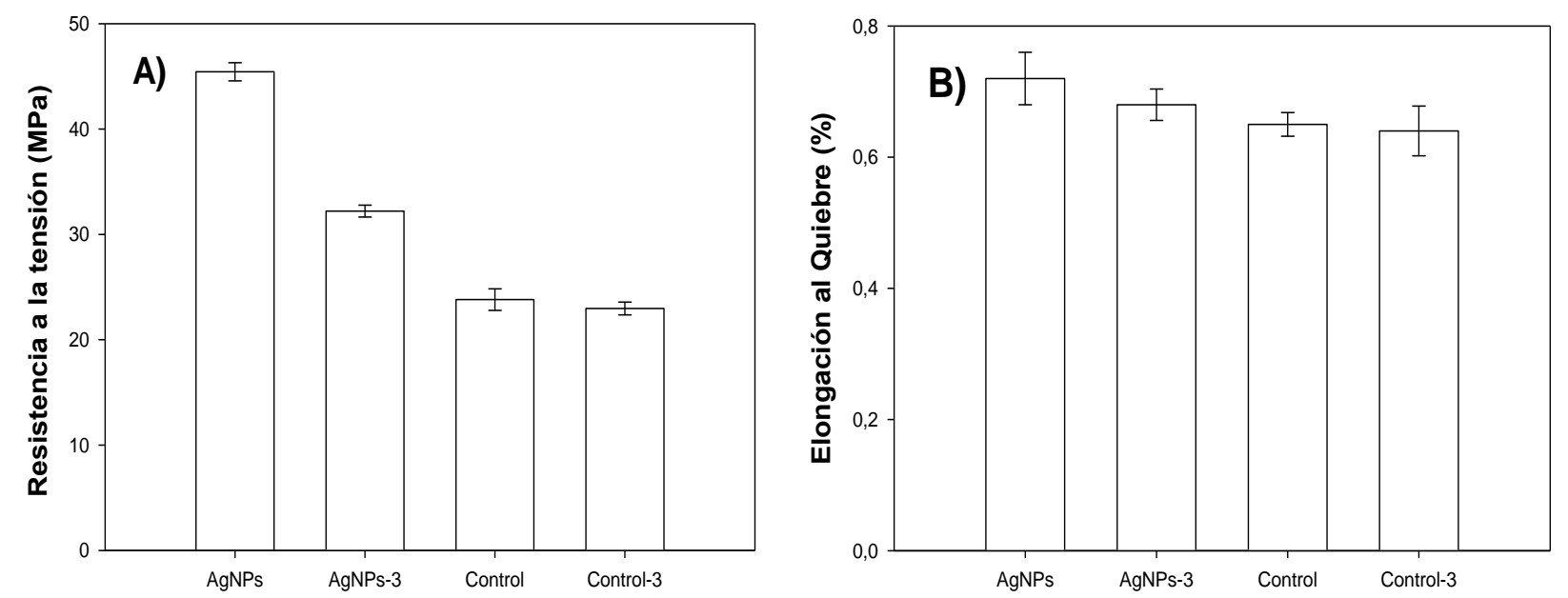

Fig. 1: Valores de Fuerza de ruptura y elongación al quiebre de biopelículas de goma gelana de bajo acilo conteniendo nanopartículas de plata. AgNPs-3 y Control-3: biopelículas con tres semanas de almacenamiento; AgNPs y Control: biopelículas al inicio del almacenamiento (semana 0) 
De forma general se puede decir que la presencia de AgNPs en las BP duplica los valores de RT, mientras que el almacenamiento ocasiona una disminución en los valores de RT. Tal comportamiento podría ser explicado por la pérdida de algunos enlaces iónicos directos entre los iones y los grupos con carga negativa presentes en las hélices de GBA (González et al., 2012), esto como consecuencia de una posible lixiviación de iones durante el almacenamiento. Con respecto a la $\mathrm{EQ}$, se observó que la presencia de AgNPs no origina cambios significativos $(P<0.05)$, debido a que al adicionar AgNPs se encontraron valores de EQ de $0.72 \%$; mientras que en las BP control este valor fue de $0.65 \%$. Es importante destacar que después del almacenamiento (tres semanas) no se presentaron pérdidas significativas $(P<0.05)$ en la flexibilidad de las BP control y aquellas BP-AgNPs. En este sentido, se podría decir que la flexibilidad no se encuentra relacionada con el tipo de ion utilizado como agente entrecruzante, ni con el tiempo de almacenamiento, pudiendo ser más relevante la estructura principal del material que da soporte a la BP. Similares resultados fueron reportados por González et al., (2017b) quienes determinaron la EQ de mezclas de BP de GAA y GBA gelificadas con iones divalentes y monovalentes, encontrando que la flexibilidad resulta mayoritariamente influenciada por el tipo de goma utilizada en comparación con los iones.

\section{Espesor, transparencia y permeabilidad al vapor de agua de las biopelículas}

Controlar el espesor de las BP es un aspecto de gran importancia en el área de empaques, debido a que esta propiedad puede llegar a modificar las propiedades tanto mecánicas como de barrera (Liling et al., 2016). En la tabla 1, se observa que las AgNPs no afectan el espesor de las BP, ya que se obtuvieron valores de 0.18 $\mathrm{mm}$ en las BP con y sin AgNPs. Igualmente, es importante considerar que tales espesores son lo suficientemente bajos para que sean calificadas como películas según ASTM 1985 (American Society for Testing and Materials (ASTM), quienes establecieron que las películas son aquellas que posean espesores menores a $0.25 \mathrm{~mm}$. En el presente estudio, el volumen para cada solución formadora de BP fue controlado durante el proceso de fabricación, con el fin de asegurar valores similares de espesor. Estos resultados son semejantes a los publicados por Mostafavi et al., (2016), quienes no encontraron diferencias significativas en el espesor de BP a base de goma tragacanto y goma algarrobo, atribuyendo tal comportamiento al estricto control ejercido durante el proceso de elaboración secado de las muestras.

La transparencia es una propiedad que requiere una atención especial en el desarrollo de BP porque afecta la apariencia en general y determina el tipo de aplicación final de la misma. En el presente trabajo, las BP con mayor transparencia fueron las BP control que presentaron índices de 3.76, mientras que las BP-AgNPs presentaron indicies de 7.86. Esto indica que la transparencia de las BP depende de la cantidad de nitrato de plata utilizada, lo cual significa que las nanopartículas disminuyen la transparencia de las BP de GBA. Esto se debe a la reducción de la plata formando las AgNPs generando una coloración amarilla parduzca, que es atribuida a la superficie característica de la resonancia del plasmón de AgNPs (Puišo et al., 2008; Zheng et al., 2001). Similares estudios fueron propuestos por Yang et al., (2010) quienes encontraron un incremento en la opacidad de BP a base de GBA al incrementar las proporciones de $\mathrm{NaCl}_{2}$. Estos autores atribuyen el aumento en la opacidad a una disminución del espacio entre cadenas poliméricas permitiendo un menor paso de la luz a través de la BP. Los valores de transparencia reportados en la presente investigación (3.76 a 7.86) son mayores a los valores publicados por Nur Hazirah et al., (2016) en BP solamente a base de CMC y gelatina (1.99). Estos resultados sugieren que las BP a base de GBA con y sin AgNPs son menos transparentes que aquellas que contienen CMC y gelatina. Después del almacenamiento, fueron obtenidos valores de transparencia de 5.43 en BP-AgNPs y de 3.57 en BP control; es decir las BP se vuelven más transparentes indicando un proceso de reducción de la plata durante el almacenamiento, y confirma que algunos iones de plata libres se liberan después de tres semanas de almacenamiento. Los iones de plata son conocidos que fácilmente se liberan en un medio ambiente reductor (Martínez-Abad, 2014).

Los valores de permeabilidad a vapor de agua (PVA) en BP con y sin AgNPs se presentan en la tabla 1, en la cual se observan diferencias significativas $(P<0.05)$ desde 10.56 hasta $13.45\left(\mathrm{~g} \mathrm{~mm} / \mathrm{m}^{2} \mathrm{~d} \mathrm{kPa}\right)$ respectivamente, estos resultados sugieren un posible incremento en el número de enlaces que contribuyen en la obtención de una red tridimensional más entretejida. Resultados similares fueron publicados por González et al., (2017b), quienes obtuvieron incrementos en los valores de PVA de 14.09 a $36.11\left(\mathrm{~g} \mathrm{~mm} / \mathrm{m}^{2} \mathrm{~d} \mathrm{kPa}\right.$ ) al incrementar las concentraciones de iones calcio y variar las proporciones de goma gelana. Después del almacenamiento las BP-AgNPs incrementan los valores de permeabilidad al vapor de agua en $2.32\left(\mathrm{~g} \mathrm{~mm} / \mathrm{m}^{2} \mathrm{~d} \mathrm{kPa}\right)$, lo cual puede suponer la pérdida de algunos enlaces como consecuencia de la lixiviación de iones de plata, aunque se necesitan más análisis con el fin de confirmar esta hipótesis. Estos resultados son contrarios a los publicados por Cano et al., (2016) quienes encontraron que los valores de PVA disminuían cuando se adicionaba plata a $\mathrm{BP}$, estos autores atribuyeron este comportamiento a las diferencias estructurales en la matriz polimérica. A bajas concentraciones de plata, las cadenas moleculares se extienden con las uniones de especies de plata, las cuales conllevan a un incremento en el factor tortuosidad para la difusión de las moléculas de agua (Cussler et al., 1998; Rhim et al., 2013). Por el contrario, mayores concentraciones de plata exhiben extensión de las macromoléculas en el medio acuoso, dando lugar a una estructura menos compacta de la BP, donde las 
moléculas de agua pueden ser transferidas rápidamente. Generalmente, la transmisión del vapor de agua a través de una BP hidrofílica, depende de la difusividad y la solubilidad de las moléculas de agua en la matriz polimérica (Gontard y Guilbert, 1994).

Tabla 1: Valores de transparencia, espesor y permeabilidad al vapor de agua de BP a base de gelana de bajo acilo. AgNPs y Control: biopelículas al inicio del almacenamiento (semana $0)$; AgNPs-3; Control-3: biopelículas con tres semanas de almacenamiento

\begin{tabular}{|c|c|c|c|}
\hline Biopelículas & Espesor $(\mathrm{mm})$ & Transparencia a $560 \mathrm{~nm}$ & $P V A\left(\mathrm{~g} \mathrm{~mm} / \mathrm{m}^{2} \mathrm{~d} \mathrm{kPa}\right)$ \\
\hline AgNPs & $0.18 \pm 0.1^{\mathrm{a}}$ & $7.86 \pm 0.2^{\mathrm{a}}$ & $13.45 \pm 0.8^{\mathrm{a}}$ \\
\hline AgNPs-3 & $0.18 \pm 0.1^{\mathrm{a}}$ & $5.43 \pm 0.2^{\mathrm{b}}$ & $15.77 \pm 0.6^{\mathrm{b}}$ \\
\hline Control & $0.18 \pm 0.1^{\mathrm{a}}$ & $3.76 \pm 0.1^{\mathrm{c}}$ & $10.56 \pm 0.5^{\mathrm{c}}$ \\
\hline Control-3 & $0.18 \pm 0.1^{\mathrm{a}}$ & $3.57 \pm 0.2^{\mathrm{c}}$ & $12.59 \pm 1.0^{\mathrm{d}}$ \\
\hline
\end{tabular}

\section{Actividad antimicrobiana}

La actividad antimicrobiana de BP control y aquellas cargadas con AgNPs fueron analizadas contra dos hongos (Aspergillus spp y Penicillium spp) y dos bacterias (Escherichia coli y Staphylococcus aureus). Las BP-AgNPs presentaron actividad antimicrobiana y anti fúngica, las cuales dependen principalmente del tipo de microorganismo evaluado. La mayor actividad antibacteriana fue observada en las BP-AgNPs después de tres semanas de almacenamiento mostrando un mayor halo de inhibición para los cuatro microorganismos evaluados. Escherichia coli resultó ser la bacteria más sensible a la presencia de AgNPs seguida por Staphylococcus aureus, mientras que los hongos presentaron similares diámetros de inhibición. Por el contrario, en las muestras control no se presentó actividad bactericida o bacteriostática frente a los microorganismos evaluados. Varios autores han encontrado que la actividad antibacteriana de las BP-AgNPs incrementa conforme aumenta la concentración de plata (Pal et al., 2007; Rai et al., 2009; Bryaskova et al., 2010; Rhim et al., 2013; Martínez-Abad et al., 2014).

La mayor sensibilidad de E. coli frente a las AgNPs es ocasionada por la composición de su pared celular en la que se encuentran lipopolisacáridos de carga negativa, que a su vez atraen los iones de plata conllevando a un incremento de la permeabilidad. En contraste, las bacterias Gram positivas ( $S$. aureus), no presentan lipopolisacáridos en su pared celular que está basada en peptidoglucano, una estructura que le confiere mayor resistencia al disminuir la penetración de AgNPs (Bryaskova et al., 2010). El mecanismo de acción de los iones de plata y las AgNPs está unido con la interacción con el grupo tiol (-SH) presente en las enzimas que intervienen en la respiración de las células bacterianas. Por ejemplo, la interacción de la plata con residuos de L- cisteína causa la desnaturalización y pérdida de las funciones enzimáticas (Feng et al., 2000; Liau et al., 1997; Martínez-Abad et al., 2014).

Cano et al., (2016) publicaron que el efecto anti fúngico de AgNPs en BP base de PVA y almidón disminuye conforme aumenta el tiempo de almacenamiento de las BP, este estudio es contrario al que aquí se reporta, en donde el afecto anti fúngico fue directamente proporcional al tiempo de almacenamiento, es decir al aumentar el tiempo de almacenamiento incremento el efecto anti fúngico como consecuencia de la mencionada lixiviación de los iones plata de la estructura de la BP. Considerando el efecto anti fúngico, hay poca información, solo Abreu et al., (2015) reportó pruebas anti fúngicas contra Candida albicans de películas cargadas con AgNPs pero no observó ningún efecto.

\section{CONCLUSIONES}

La utilización de nanopartículas de plata en la elaboración biopelículas a base de gelana de bajo acilo puede ser útil en la producción de recubrimientos activos con mejores propiedades mecánicas y antimicrobianas. Aunque el tiempo de almacenamiento logra disminuir la resistencia a la tensión, también potencializa el efecto antimicrobiano, lo cual puede ser consecuencia de un proceso de lixiviación de los iones plata que al liberarse producen una disminución en el número de enlaces presentes en la matriz polimérica y ocasionan daños principalmente en las bacterias Gram. Estos resultados son prometedores y apuntan a la posibilidad de utilizar biopelículas de goma gelana de bajo acilo como sistema de vehiculización de AgNPs para su liberación gradual manteniendo un efecto antimicrobiano sostenido. Aunque más estudios son necesarios con el fin de mejorar las propiedades físico mecánicas que aquí se reportan. 


\section{REFERENCIAS}

Abreu, A. y otros cinco autores, Antimicrobial nanostructured starch based films for packaging, Carbohydrate Polymers, 129, 127-134 (2015)

American Society for Testing and Materials (ASTM), Standard terminology relating to plastic, Designation D883-00, Annual book of ASTM standards, Philadelphia, 13 (1985)

Bryaskova, R. y otros cuatro autores, Synthesis, characterisation and antibacterial activity of PVA/TEOS/AgNp hybrid thin films, Journal of Colloid and Interface Science, 349, 77-85 (2010)

Cano, A. y otros tres autores, Development and characterization of active films based on starch-PVA, containing silver nanoparticles, Food Packaging and Shelf Life, 10, 16-24 (2016)

Corrales, M., A. Fernández y J. Han, Antimicrobial packaging systems, In J.H. Han (Ed.), Innovations in food packaging (pp. 133-170), $2^{\text {nd }}$ Ed., San Diego, CA, USA: Academia Press (2014)

Cussler, E. y otros tres autores, Barrier membranes, Journal of Membrane Science, 38, 161-174 (1998)

Feng, y otros cinco autores, A mechanistic study of the antibacterial effect of silver ions on Escherichia coli and Staphylococcus aureus, Journal of Biomedical Materials Research, 52(4), 662-668 (2000)

Ghosh, S. y otros seis autores, Antimicrobial activity of highly stable silver nanoparticles embedded in agaragar matrix as a thin film, Carbohydrate Research, 345, 2220-2227 (2010)

Gontard, N. y S. Guilbert, Biopackaging: technology and properties of edible and/or biodegradable material of agricultural origin. In M. Mathlouthi, Food packaging and preservation. London: Blackie Academic \& Professional (1994)

González, R. y otros tres autores, Rheological characterization and activation energy values of binary mixtures of gellan, European Food Research Technology, 235, 305-313 (2012)

González, R. y otros dos autores, Efecto de la Adición de Goma Gelana sobre las Propiedades de Barrera y Físico Mecánicas de Biopelículas Binarias, Información Tecnológica, 28(1), 43-150 (2017a)

González, R. y otros dos autores, Incidencia de los lones Divalentes y Monovalentes en las Propiedades Físico Mecánicas y de Barrera en Biopelículas a Base de Goma Gelana, Información Tecnológica, 28(2), 67$74(2017 b)$

Kanmani, P. y J. Rhim, Physicochemical properties of gelatin/silver nanoparticle antimicrobial composite films, Food Chemistry, 148 (1), 162-169 (2014)

Kokoszka, S., Debeaufort, F. y otros tres autores, Protein and glycerol contents affect physico-chemical properties of soy protein isolate-based edible films, Innovative Food Science \& Emerging Technologies, 11, 503-510 (2010)

Kurt, A. y T. Kahyaoglu, Characterization of a new biodegradable edible film made from salep glucomannan, Carbohydrate Polymers, 104(0), 50-58 (2014)

Liau, S. y otros cuatro autores, Interaction of silver nitrate with readily identifiable groups: Relationship to the antibacterial action of silver ions, Letters in Applied Microbiology, 25(4), 279-283 (1997)

Liling, G. y otros cinco autores, Effects of ionic crosslinking on physical and mechanical properties of alginate mulching films, Carbohydrate Polymers, 136, 259-265 (2016)

Lin, S. y otros cinco autores, Novel antimicrobial chitosan-cellulose composite films bioconjugated with silver nanoparticles, Industrial Crops and Products, 70, 395-403 (2015)

Martínez-Abad, A. y otros tres autores, On the different growth conditions affecting silver antimicrobial efficacy on Listeria monocytogenes and Salmonella entérica, Inter. Journal of Food Microbiology, 158(2), 147-154 (2012)

Martínez-Abad, A. Tesis: Development of silver based antimicrobial films for coating and food packaging applications. Directed by: Ocio, M.J., y Lagarón, J.M., U. Politénica de Valencia. España, Febrero (2014) 
Martínez-Abad, A., J. Lagarón y M. Ocio, Antimicrobial beeswax coated polylactide films with silver control release capacity, International Journal of Food Microbiology, 174, 39-46 (2014)

Metak, A., F. Nabhani y N. Connolly, Migration of engineered nanoparticles from packaging into food products. LWT-Food Science and Technology, 64, 781-787 (2015)

Monge, M., Nanopartículas de plata: Métodos de síntesis en disolución y propiedades bactericidas. Real Sociedad Española de Química, Anales de Química, 105(1), 33-41 (2009)

Mostafavi, S. y otros tres autores, Preparation and characterization of tragacanth-locust bean gum edible blend films, Carbohydrate Polymers, 139 (2), 20-27 (2016)

Nur Hazirah, M., N. Isab y M. Sarbona, Effect of xanthan gum on the physical and mechanical properties of gelatin-carboxymethyl cellulose film blends, Food Packaging and Shelf Life, 9, 55-63 (2016)

Pal, S., Y. Tak y J. Song, Does the antibacterial activity of silver nanoparticles depend on the shape of the nanoparticle? A study of the Gram-negative bacterium Escherichia coli, Applied and Environmetal Microbiology, 73, 1712-1720 (2007)

Puišo, J. y otros tres autores, Plasmonic properties of silver in polymer. Materials Science and Engineering: B, 149, 230-236 (2008)

Rai, M., A. Yadav y A. Gade, Silver nanoparticles as a new generation of antimicrobials, Biotechnology Advances, 27, 76-83 (2009)

Rhim, J., L. Wang y S. Hong, Preparation and characterization of agar/ silver nanoparticles composite films with antimicrobial activity, Food Hydrocolloids, 327-335 (2013)

Shameli, K. y otros seis autores, Silver/poly (lactic acid) nanocomposites: Preparation, characterization, and antibacterial activity, International Journal of Nanomedicine, 5, 573-579 (2010)

Sedlarik, V. y otros cuatro autores, The effect of preparation temperature on the mechanical and antibacterial properties of poly (vinyl alcohol)/silver nitrate films, Polymer Degradation and Stability, 95, 399-404 (2010)

Vimala, K. y otros seis autores, Fabrication of curcumin encapsulated chitosan-PVA silver nanocomposite films for improved antimicrobial activity, Journal of Biomaterials and Nanobiotechnology, 2, 55-64 (2011)

Yang, L., A. Paulson y M. Nickerson, Mechanical and physical properties of calcium-treated gellan films, Food Research International, 43, 1439-1443 (2010)

Zheng, R. y otros cuatro autores, Interfacial interaction in Ag/polymer nanocomposite films, Journal of Materials Science Letters, 20, 1473-1476 (2001) 